$12-1-2008$

\title{
Scattering of an Electromagnetic Plane Wave by a Luneburg Lens. II. Wave Theory
}

James A. Lock

Cleveland State University, j.lock@csuohio.edu

Follow this and additional works at: https://engagedscholarship.csuohio.edu/sciphysics_facpub

Part of the Physics Commons

How does access to this work benefit you? Let us know!

\section{Publisher's Statement}

This paper was published in Journal of the Optical Society of America A: Optics Image Science and Vision and is made available as an electronic reprint with the permission of OSA. The paper can be found at the following URL on the OSA website: http://www.opticsinfobase.org/josaa/ abstract.cfm?URI=josaa-25-12-2980. Systematic or multiple reproduction or distribution to multiple locations via electronic or other means is prohibited and is subject to penalties under law.

\section{Original Citation}

Lock, James A. "Scattering of an Electromagnetic Plane Wave by a Luneburg Lens. II. Wave Theory." Journal of the Optical Society of America A: Optics Image Science and Vision 25 (2008): 2980-2990.

\section{Repository Citation}

Lock, James A., "Scattering of an Electromagnetic Plane Wave by a Luneburg Lens. II. Wave Theory" (2008). Physics Faculty Publications. 31.

https://engagedscholarship.csuohio.edu/sciphysics_facpub/31

This Article is brought to you for free and open access by the Physics Department at EngagedScholarship@CSU. It has been accepted for inclusion in Physics Faculty Publications by an authorized administrator of EngagedScholarship@CSU. For more information, please contact library.es@csuohio.edu. 


\title{
Scattering of an electromagnetic plane wave by a Luneburg lens. II. Wave theory
}

\author{
James A. Lock \\ Department of Physics, Cleveland State University, Cleveland, Ohio 44115, USA (j.lock@csuohio.edu)
}

Received July 30, 2008; accepted September 11, 2008;

posted September 29, 2008 (Doc. ID 99458); published November 12, 2008

\begin{abstract}
The partial wave scattering and interior amplitudes for the interaction of an electromagnetic plane wave with a modified Luneburg lens are derived in terms of the exterior and interior radial functions of the scalar radiation potentials evaluated at the lens surface. A Debye series decomposition of these amplitudes is also performed and discussed. The effective potential inside the lens for the transverse electric polarization is qualitatively examined, and the approximate lens size parameters of morphology-dependent resonances are determined. Finally, the physical optics model is used to calculate wave scattering in the vicinity of the ray theory orbiting condition in order to demonstrate the smoothing of ray theory discontinuities by the diffraction of scattered waves. (C) 2008 Optical Society of America
\end{abstract} OCIS codes: $050.1960,080.2710,290.4020$.

\section{INTRODUCTION}

This is the second in a series of papers whose purpose is to examine electromagnetic scattering by a modified Luneburg lens as a prototype example for understanding the scattering phenomena of a radially inhomogeneous sphere and to compare and contrast them to the way in which they occur for scattering by a homogeneous sphere. The first paper [1] considered ray theory transmission through a sphere of radius $a$ whose dielectric constant $N^{2}(r)$ varies parabolically in $r$ in such a way that the focal point of all the transmitted rays is $f a$. Such a sphere is called a modified Luneburg lens. A discontinuity was found as a function of $f$ in the trajectory of the ray with grazing incidence on the sphere. For $f<1$, the grazing incidence ray was scattered through $\theta=180^{\circ}$, for $f=1$ it was scattered through $\theta=90^{\circ}$, and for $f>1$ it was scattered through $\theta=0^{\circ}$. Similarly, there was a discontinuity in the scattered intensity of the grazing incident ray. For $f>1$ the intensity diverged as $f \rightarrow 1$ due to the transmission rainbow approaching $\theta=90^{\circ}$. But for $f=1$, the intensity at $\theta=90^{\circ}$ vanished. Both of these discontinuities signal a nonuniform convergence of ray theory in the vicinity of the grazing incidence ray.

In this paper the ray theory discontinuities are smoothed by diffractive effects when scattering by light waves is considered. In Section 2, an incident plane wave is decomposed into partial waves. The scattering of each partial wave by a sphere having an arbitrary radially inhomogeneous refractive index profile is determined in terms of the solutions of the second-order differential equation for the radial functions of the transverse electric (TE) and transverse magnetic (TM) scalar radiation potentials. For TE scattering by a modified Luneburg lens, the radial functions are related to Whittaker functions $[2,3]$. In order to obtain an intuitive understanding of a number of the resulting scattering phenomena, in Section 3 the differential equation for the TE partial wave radial function is qualitatively analyzed using the effective po- tential approach borrowed from quantum mechanics [4]. It is found that for $f=1$, scattering of the grazing incidence ray corresponds to the semiclassical phenomenon of orbiting [5,6]. For $f<1$, the effective potential has a wide, shallow well inside the modified Luneburg lens that can support a series of morphology-dependent resonances (MDRs). The approximate size parameter of the resonances is derived as a function of partial wave number and radial mode number. In Section 4, the physical optics model for scattering of the grazing incident ray is developed for $f=1$, and the smoothing of the ray theory discontinuities at the orbiting condition by wave diffraction is demonstrated and physically interpreted. Finally, Appendix A gives the details of orbiting for classical scattering of a particle by a force center. Appendix B derives the Debye series expansion of the partial wave scattering and interior amplitudes for a sphere with an arbitrary radially inhomogeneous refractive index profile. This Debye series has a slightly different physical interpretation than it does for a homogeneous sphere, which is commented on in Appendix B as well. The results obtained here and in [1] are numerically tested in [7] where a modified Luneburg lens is approximated by a finely stratified multilayer sphere. In [7] also, a new algorithm for computing scattering by a multilayer sphere based on an analogy to the successive doubling strategy of the fast Fourier transform (FFT) algorithm is developed and implemented.

\section{FORMAL SOLUTION TO THE WAVE SCATTERING PROBLEM}

\section{A. Radial Wave Equations}

In analogy to the procedure followed in Mie theory for scattering of a plane wave by a homogeneous sphere, the TE and TM fields inside a sphere having the arbitrary refractive index profile $N(r)$ are obtained by taking various vector derivatives of the scalar radiation potentials. For 
the TE polarization, a partial wave scalar radiation potential inside the sphere is of the form

$$
\psi_{n, m}(k r, \theta, \varphi)=\left[F_{n}(k r) / k r\right] P_{n}^{m}[\cos (\theta)] \exp (\operatorname{im} \varphi),
$$

where $\lambda$ is the wavelength of the incident electromagnetic wave in vacuum, the wavenumber is $k=2 \pi / \lambda$, the partial wavenumber is $n$, the azimuthal mode number is $m$, and $P_{n}{ }^{m}$ are associated Legendre polynomials. Equation (1) satisfies the wave equation

$$
\nabla^{2} \psi_{n, m}+N^{2}(r) k^{2} \psi_{n, m}(k r)=0,
$$

which reduces to [8-11]

$$
\mathrm{d}^{2} F_{n} / \mathrm{d}(k r)^{2}+\left\{N^{2}(r)-n(n+1) /(k r)^{2}\right] F_{n}(k r)=0
$$

for the partial wave radial function $F_{n}(k r)$. If the refractive index $N$ is constant, $F_{n}(k r)$ is a linear combination of Riccati-Bessel functions $\psi_{n}(N k r)=N k r j_{n}(N k r)$ and Riccati-Neumann functions $\chi_{n}(N k r)=N k r n_{n}(N k r)$, where $j_{n}$ and $n_{n}$ are spherical Bessel functions and spherical Neumann functions, respectively. The partial wave TE electric and magnetic fields associated with this radiation potential are

$$
\begin{aligned}
& \mathbf{E}_{n, m}{ }^{\mathrm{TE}}(r, \theta, \varphi)=-\mathbf{r} \times \nabla \psi_{n, m}, \\
& \mathbf{B}_{n, m}{ }^{\mathrm{TE}}(r, \theta, \varphi)=(i / \omega) \nabla \times\left(\mathbf{r} \times \nabla \psi_{n, m}\right),
\end{aligned}
$$

where the speed of light in vacuum is $c$ and $\omega=c k$.

For the TM polarization, a partial wave scalar radiation potential is of the form

$$
\begin{aligned}
\xi_{n, m}(k r, \theta, \varphi) & =N(r) \psi_{n, m}(k r, \theta, \varphi) \\
& =\left[G_{n}(k r) / k r\right] P_{n}{ }^{m}(\cos \theta) \exp (\operatorname{im} \varphi)
\end{aligned}
$$

and satisfies the wave equation

$$
\begin{aligned}
& \nabla^{2} \xi_{n, m}-[2(\mathrm{~d} N / \mathrm{d} r) / N] \partial \xi_{n, m} / \partial r \\
& \quad-[2(\mathrm{~d} N / \mathrm{d} r) /(N r)] \xi_{n, m}+N^{2}(r) k^{2} \xi_{n, m}=0 .
\end{aligned}
$$

It reduces to [8-11]

$$
\begin{array}{r}
\mathrm{d}^{2} G_{n} / \mathrm{d}(k r)^{2}-2(\mathrm{~d} N / \mathrm{d} r)\left[\mathrm{d} G_{n} / \mathrm{d}(k r)\right] / N k \\
+\left[N^{2}(r)-n(n+1) /(k r)^{2}\right] G_{n}(k r)=0
\end{array}
$$

for the partial wave radial function $G_{n}(k r)$. If the refractive index $N$ is constant, $G_{n}(k r)$ is again a linear combination of $\psi_{n}(N k r)$ and $\chi_{n}(N k r)$. The partial wave TM electric and magnetic fields associated with this radiation potential are

$$
\begin{aligned}
& \mathbf{E}_{n, m}{ }^{\mathrm{TM}}(r, \theta, \varphi)=\left(i c / N^{2} \omega\right) \nabla \times\left(\mathbf{r} \times \nabla \xi_{n, m}\right), \\
& \mathbf{B}_{n, m}{ }^{\mathrm{TM}}(r, \theta, \varphi)=(1 / c) \mathbf{r} \times \nabla \xi_{n, m} .
\end{aligned}
$$

The structure of the differential equation for the TE radial function $F_{n}$ of Eq. (3) is exactly as it was for a homogeneous sphere, except that now the refractive index is a function of $r$. The differential equation for the TM radial function $G_{n}$ of Eq. (7) contains a first derivative term proportional to $\mathrm{d} N / \mathrm{d} r$, which greatly complicates its analytical solution.

\section{B. Boundary Condition Matching}

Consider a plane wave with electric field strength $E_{0}$, traveling in the positive $z$ direction in the external medium and polarized in the $x$ direction. It is incident on a radially inhomogeneous sphere of radius $a$ and arbitrary refractive index profile $N(r)$ whose center is at the origin of coordinates. The complete scalar radiation potential $\Psi$ of the TE and TM components of the plane wave contains only the $m= \pm 1$ azimuthal modes and is

$$
\begin{aligned}
\Psi_{\text {inc }}(k r, \theta, \varphi)= & \sum_{n=1}^{\infty}\left\{i^{n}(2 n+1) /[n(n\right. \\
& +1)]\} \psi_{n}(k r) P_{n}{ }^{1}(\cos \theta) \alpha(\varphi),
\end{aligned}
$$

where

$$
\begin{array}{rlr}
\alpha(\varphi) & =\cos (\varphi) & \text { for } \mathrm{TM} \\
& =\sin (\varphi) & \text { for } \mathrm{TE} .
\end{array}
$$

Similarly, the complete scalar radiation potential of the scattered wave in the exterior medium is

$$
\begin{aligned}
\Psi_{\text {scat }}(k r, \theta, \varphi)= & -\sum_{n=1}^{\infty}\left\{i^{n}(2 n+1) /[n(n+1)]\right\} \\
& \times \zeta_{n}{ }^{(1)}(k r) P_{n}{ }^{1}(\cos \theta) \beta_{n}(\varphi),
\end{aligned}
$$

where $\zeta_{n}{ }^{(1)}(k r)=k r h_{n}{ }^{(1)}(k r)$ are radially outgoing RiccatiHankel functions,

$$
\begin{aligned}
\beta_{n}(\varphi)=a_{n} \cos (\varphi) & \text { for TM } \\
=b_{n} \sin (\varphi) & \text { for TE }
\end{aligned}
$$

and $a_{n}$ and $b_{n}$ are the partial wave scattering amplitudes. The complete interior scalar radiation potential is

$$
\Psi_{\mathrm{int}}(k r, \theta, \varphi)=\sum_{n=1}^{\infty}\left\{i^{n}(2 n+1) /[n(n+1)]\right\} \gamma_{n}(k r, \varphi) P_{n}{ }^{1}(\cos \theta),
$$

where

$$
\begin{aligned}
\gamma_{n}(k r, \varphi) & =G_{n}(k r) c_{n} \cos (\varphi) \quad \text { for TM, } \\
& =F_{n}(k r) \mathrm{d}_{n} \sin (\varphi) \quad \text { for TE }
\end{aligned}
$$

where $G_{n}(k r)$ and $F_{n}(k r)$ are the solutions of Eqs. (3) and (7), respectively, that vanish at the origin and $c_{n}$ and $d_{n}$ are the partial wave interior amplitudes. Then continuity of $\mathbf{D}_{\mathrm{rad}}, \mathbf{E}_{\mathrm{tan}}$, and $\mathbf{B}_{\tan }$ at $r=a$ gives [8-11]

$$
\begin{aligned}
a_{n}= & {\left[G_{n}{ }^{\prime}(k a) \psi_{n}(k a)-N^{2}(a) G_{n}(k a) \psi_{n}{ }^{\prime}(k a)\right] /\left[G_{n}{ }^{\prime}(k a)\right.} \\
& \left.\times \zeta_{n}{ }^{(1)}(k a)-N^{2}(a) G_{n}(k a) \zeta_{n}{ }^{(1) \prime}(k a)\right], \\
b_{n}= & {\left[F_{n}{ }^{\prime}(k a) \psi_{n}(k a)-F_{n}(k a) \psi_{n}{ }^{\prime}(k a)\right] /\left[F_{n}{ }^{\prime}(k a) \zeta_{n}{ }^{(1)}(k a)\right.} \\
& \left.-F_{n}(k a) \zeta_{n}{ }^{(1) \prime}(k a)\right], \\
c_{n}= & -i N^{2}(a) /\left[G_{n}{ }^{\prime}(k a) \zeta_{n}{ }^{(1)}(k a)-N^{2}(a) G_{n}(k a) \zeta_{n}{ }^{(1) \prime}(k a)\right],
\end{aligned}
$$




$$
\mathrm{d}_{n}=-i /\left[F_{n}{ }^{\prime}(k a) \zeta_{n}^{(1)}(k a)-F_{n}(k a) \zeta_{n}{ }^{(1) \prime}(k a)\right] .
$$

In Eqs. (15a)-(15d) the prime symbol indicates a derivative with respect to $k r$, and $N(a)$ is evaluated inside the sphere as $r \rightarrow a$. These partial wave scattering and interior amplitudes are then substituted into Eqs. (11) and (13), from which the scattered and interior fields can be determined. This constitutes the formal solution to the electromagnetic boundary value problem of a plane wave scattered by a radially inhomogeneous sphere. The solution is formal in the sense that the radial functions $F_{n}$ and $G_{n}$ still need to be determined for a given refractive index profile, and the infinite series of partial waves needs to be summed in order to identify and understand various features of the scattered intensity.

\section{Wave Scattering by a Modified Luneburg Lens}

A modified Luneburg lens has the refractive index profile [1]

$$
N(r)=\left[1+f^{2}-(r / a)^{2}\right]^{1 / 2} / f .
$$

Equation (3) for the TE scalar radiation potential $F_{n}(k r)$ then becomes

$$
\begin{aligned}
& \mathrm{d}^{2} F_{n} / \mathrm{d}(k r)^{2}+\left[-n(n+1) /(k r)^{2}+\left(f^{2}+1\right) / f^{2}-(k r)^{2} /(f k a)^{2}\right] F_{n} \\
& \quad=0
\end{aligned}
$$

whose solution, after a change of variables, is the Whittaker function $[2,3]$

$$
\begin{aligned}
F_{n}(k r)= & {\left[(k r)^{2} / f k a\right]^{(n+1) / 2} \exp \left[-(k r)^{2} / 2 f k a\right] \times M[(2 n+3) / 4} \\
& \left.-\left(f^{2}+1\right) k a / 4 f,(2 n+3) / 2 ;(k r)^{2} / f k a\right],
\end{aligned}
$$

where $M(a, c ; w)$ is a confluent hypergeometric function. In the context of quantum mechanics, Eqs. (17) and (18) are identical to the radial Schrodinger equation for the three-dimensional harmonic oscillator potential in spherical coordinates and its solution, with the replacement $\left(f^{2}+1\right)(k a) / 4 f \rightarrow(E / 2 \hbar)(m / k)^{1 / 2}$. For the TM polarization, Eq. (7) for the scalar radiation potential $G_{n}(k r)$ becomes

$$
\begin{aligned}
\mathrm{d}^{2} G_{n} / \mathrm{d}(k r)^{2}+\left[2 k r /(f k a)^{2}\right]\left[\left(f^{2}+1\right) / f^{2}\right. \\
\left.\quad-(k r / f k a)^{2}\right]^{-1} \mathrm{~d} G_{n} / \mathrm{d}(k r)+\left[-n(n+1) /(k r)^{2}+\left(f^{2}+1\right) / f^{2}\right. \\
\left.\quad-(k r)^{2} /(f k a)^{2}\right] G_{n}=0 .
\end{aligned}
$$

The analytical solution of this equation [12] is considerably more complicated than that of Eq. (17).

\section{EFFECTIVE POTENTIAL APPROACH FOR TE SCATTERING}

\section{A. General Considerations}

Since the radial functions $F_{n}(k r)$ and $G_{n}(k r)$ of Eqs. (17) and (19) for a modified Luneburg lens are both complicated and relatively unfamiliar, two procedures may be used to gain intuition concerning the behavior of these functions: (i) analyzing the sphere's effective potential well and (ii) approximating the radially inhomogeneous refractive index profile by a finely stratified multilayer sphere and then numerically solving the multilayer sphere scattering problem. In this section the effective po- tential approach is discussed for TE scattering. The finely stratified sphere model is implemented and discussed in [7].

The differential equation of Eq. (17) for $F_{n}(k r)$ for the modified Luneburg lens can be rewritten as

$$
-\mathrm{d}^{2} F_{n} / \mathrm{d}(k r)^{2}+U_{\text {eff }}(k r) F_{n}(k r)=F_{n}(k r),
$$

where the effective potential is

$$
\begin{array}{rlr}
U_{\mathrm{eff}}(k r) & =n(n+1) /(k r)^{2}+\left[-1+(k r)^{2} /(k a)^{2}\right] / f^{2} & \text { for } r \leqslant a, \\
& =n(n+1) /(k r)^{2} \quad \text { for } r>a .
\end{array}
$$

Equations (20) and (21) are analogous to a onedimensional quantum mechanical Schrodinger equation [4] with wave function $F_{n}(k r)$, with unit energy, and with the potential well $U_{\text {eff }}(k r)$. The effective potential for $r \leqslant a$ is the sum of the centrifugal potential proportional to $1 / r^{2}$ and a harmonic oscillator potential proportional to $r^{2}$. Only the centrifugal potential occurs outside the sphere, and $U_{\text {eff }}$ is continuous at $r=a$. As the partial wave number $n$ increases for constant $k a$, the effective energy remains constant and the centrifugal barrier becomes higher. The radial interval for which $U_{\text {eff }}<1$ corresponds to a classically allowed region where $F_{n}(k r)$ is oscillatory, and the radial interval for which $U_{\text {eff }}>1$ corresponds to a classically forbidden region where $F_{n}(k r)$ is damped. The second derivative of $F_{n}(k r)$ vanishes at the boundary between these two regions and is called a classical turning point. For the remainder of this section, the partial wave number $n$ is parameterized by $X$, where

$$
X \equiv n(n+1) /(k a)^{2} .
$$

The effective potential approach cannot be applied to the TM partial wave radial function because of the presence of the first derivative term in Eq. (19).

\section{B. Modified Luneburg Lens with $\boldsymbol{f}>1$}

The shape of $U_{\text {eff }}(k r)$ for the TE polarization is shown for a number of values of the partial wave $X$ for $f>1$ in Fig. 1(a), for $f=1$ in Fig. 1(b), and for $f<1$ in Fig. 1(c). For $f>1$ and low partial waves corresponding to $X<1$, the classically allowed region inside the modified Luneburg lens is

$$
\left\{\left(f^{2}+1\right)-\left[\left(f^{2}+1\right)^{2}-4 f^{2} X\right]^{1 / 2}\right\} / 2 \leqslant(k r / k a)^{2} \leqslant 1 .
$$

A partial wave with $n<k a$ is incident on the lens surface and penetrates into it until the centrifugal barrier's increasing strength converts the wave from oscillatory to evanescent, after which it tunnels to the origin. As the partial wave penetrates into the lens for some distance, its oscillatory shape is distorted and its phase is shifted by the refractive index variation, and it thus contributes to the scattered intensity. The localization principle of van de Hulst associates a partial wave with the impact parameter of an incident ray, $\sin (\beta)$, and is written as

$$
X \approx \sin ^{2}(\beta)
$$

in the notation of Eq. (22) and [1]. Substituting Eq. (24) into the left side of Eq. (23), the classical turning point of the partial wave radial function inside the lens is found to be identical to the distance of closest approach of the cor- 

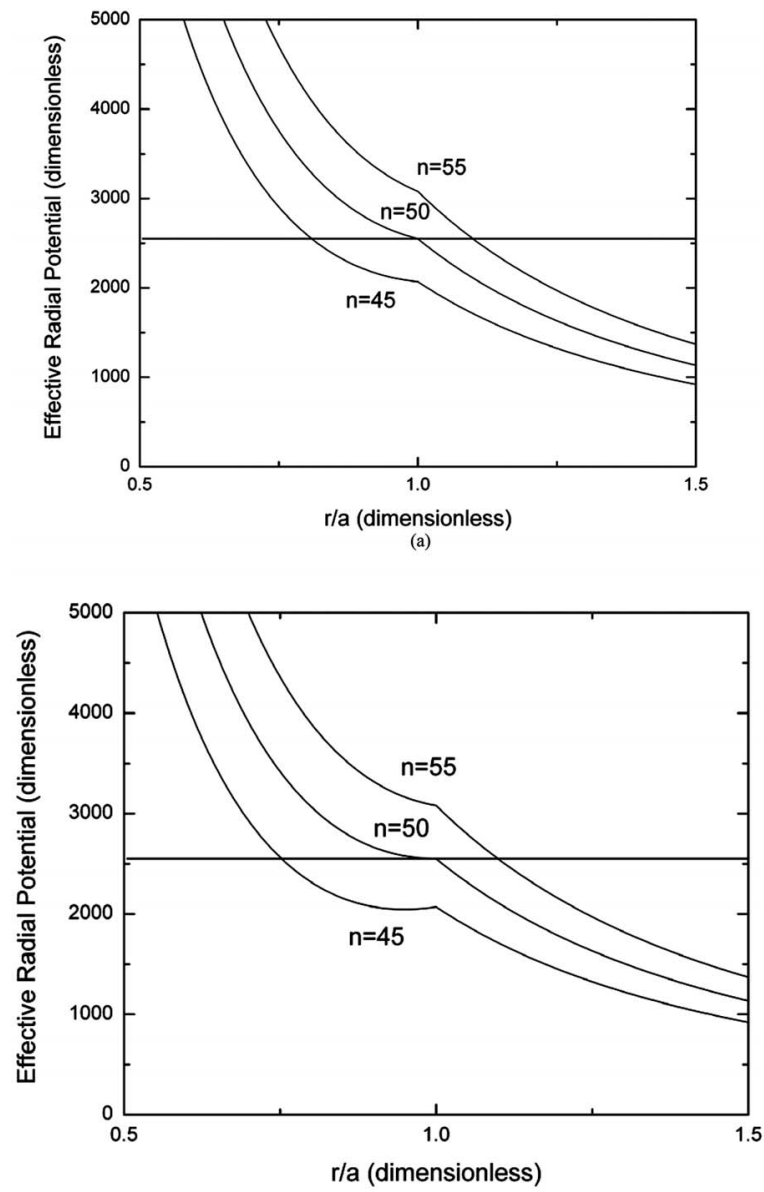

(b)

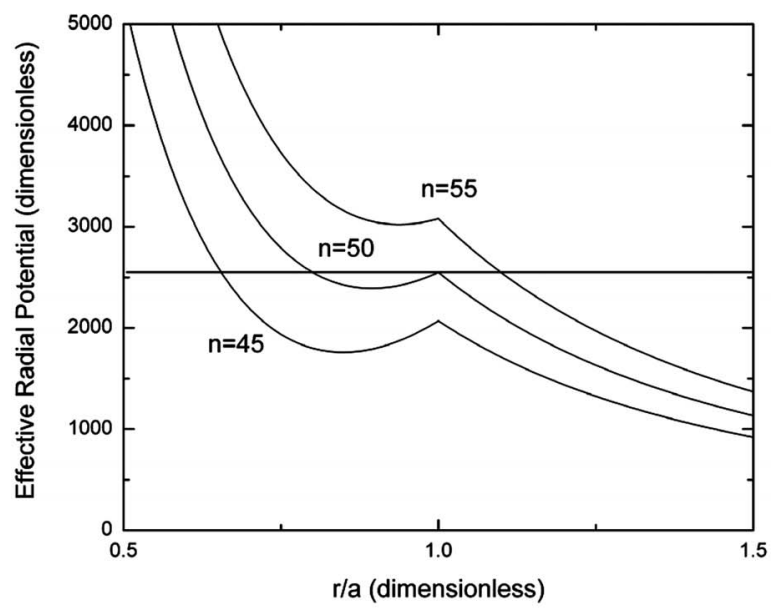

(c)

Fig. 1. Effective radial potential of Eq. (21) as a function of $r / a$ for $k a=50.5$, partial waves $n=45,50$, and 55 , and (a) $f=1.2$, (b) $f=1.0$, and (c) $f=0.8$. The effective energy of the size parameter $k a=50.5$ is the horizontal line $U_{\text {eff }}=2550$. For $f=0.8$, an internal well potential is formed by partial waves with $n$ slightly larger than 50. For $f=1$, the partial wave $n=50$ is at the condition for orbiting.

responding ray to the origin obtained in Eq. 24 of Ref. [1], further reinforcing the correspondence between the radial propagation of partial waves and the curved trajectories of rays.

When the partial wave number increases to $X=1$, the classically allowed region ends at $r=a$, the slope of $U_{\text {eff }}$ in- side the sphere is negative as $r \rightarrow a$, and the partial wave radial function is evanescent for the entire lens interior. This is shown in Fig. 1(a). This higher partial wave cannot effectively probe the details of the lens interior, and thus its contribution to the scattered intensity is small. When the partial wave number further increases to $X$ $>1$, the contribution to the scattered field rapidly decreases as the centrifugal barrier becomes increasingly high and the damping rate of the evanescent wave inside the sphere becomes progressively faster.

C. Classical Orbiting in a Luneburg Lens with $\boldsymbol{f}=\mathbf{1}$ When $f=1$, the effective potential is

$$
\begin{aligned}
U_{\mathrm{eff}}(k r) & =X(k a / k r)^{2}+(k r / k a)^{2}-1 \quad \text { for } r \leqslant a, \\
& =X(k a / k r)^{2} \quad \text { for } r>a .
\end{aligned}
$$

For low partial waves with $X<1$, the classically allowed region of the lens interior is again given by Eq. (23), and for high partial waves with $X>1$ the interior is a classically forbidden region that produces minimal scattering. For the partial wave corresponding to $X=1$, the classical turning point is at $r=a$, and the first derivative of $U_{\text {eff }}$ vanishes inside the sphere as $r \rightarrow a$. This is shown in Fig. 1(b). However, the derivative does not vanish outside the sphere as $r \rightarrow a$, since only the monotonically decreasing centrifugal potential contributes to $U_{\text {eff }}$ there. As is described in Appendix A, the phenomenon of orbiting $[5,6]$ in classical scattering occurs for a particle in an attractive potential when the first derivative of $U_{\text {eff }}$ vanishes at the classical turning point, e.g., when $U_{\text {eff }}$ is locally quadratic and the turning point occurs at the relative maximum of $U_{\text {eff. }}$ When this condition is met, a classical particle approaching the force center will not be scattered by it but will be captured by the force center and orbit it forever. It should be noted that the particular geometry of a Luneburg lens permits the orbiting condition to be met at the relative minimum of the parabolic potential, as in Fig. 1 (b), instead of the relative maximum. Although the Luneburg lens effective potential for $X=1$ does not precisely correspond to the condition for classical orbiting, since the first derivative of $U_{\text {eff }}$ at the classical turning point vanishes only as $r$ increases toward $a$, it is about as close to it as a relatively simple optical scattering geometry can come. If a classical particle is incident on the force center with slightly different initial conditions, it will be temporarily captured by the force center, orbit it for a number of cycles, and then eventually escape. The optical analog of temporary capture is closely approximated by an MDR [13].

As was seen in [1], the geometrical ray corresponding to the $X=1$ partial wave strikes the lens surface with grazing incidence and travels in a circular arc on the surface for a quarter cycle before breaking free and scattering at $90^{\circ}$. But intuitively, once the initially grazing ray is traversing the lens surface, there appears to be no special physical constraint that would limit the ray to orbit for only a quarter cycle. It could equally well traverse the lens surface for any number of orbits, thus being the optical analog of an orbiting particle in classical scattering 
[14]. In Section 4 this phenomenon is analyzed more quantitatively using the physical optics model.

\section{Morphology-Dependent Resonances of a Modified Luneburg Lens with $f<1$}

For $f<1$ and low partial waves with $X<1$, the classically allowed region inside the modified Luneburg lens is again given by Eq. (23). For $X=1$, the slope of $U_{\text {eff }}$ inside the sphere is positive as $r \rightarrow a$, signaling the presence of a relative minimum of $U_{\text {eff }}$ for $r<a$. This is apparent in Fig. 1(c). For partial waves $X \geqslant 1$ with

$$
1 \leqslant X \leqslant\left[\left(f^{2}+1\right) / 2 f\right]^{2}
$$

corresponding to geometrical rays that just miss striking the lens surface, a classically allowed well region is formed inside the lens that extends from

$$
\begin{aligned}
& \left\{\left(f^{2}+1\right)-\left[\left(f^{2}+1\right)^{2}-4 f^{2} X\right]^{1 / 2}\right\} / 2 \\
& \quad \leqslant(k r / k a)^{2} \leqslant\left\{\left(f^{2}+1\right)+\left[\left(f^{2}+1\right)^{2}-4 f^{2} X\right]^{1 / 2}\right\} / 2 .
\end{aligned}
$$

In order to get to this interior well region, the partial wave must tunnel through the centrifugal barrier, which extends from

$$
\left\{\left(f^{2}+1\right)+\left[\left(f^{2}+1\right)^{2}-4 f^{2} X\right]^{1 / 2}\right\} / 2 \leqslant(k r / k a)^{2} \leqslant X .
$$

Equation (26) defines the so-called edge region for scattering by a modified Luneburg lens with $f<1$, and MDRs should occur when partial waves in this interval are resonantly captured by the interior well. Using Eq. (15b), this occurs when

$$
F_{n}{ }^{\prime}(k a) / F_{n}(k a)=\chi_{n}{ }^{\prime}(k a) / \chi_{n}(k a) .
$$

A simple approximation for the values of the resonant size parameter for a given partial wave in the edge region can be derived as follows. When $U_{\text {eff }}$ is Taylor series expanded about its relative minimum, the resulting well

$$
U_{\mathrm{eff}}(k r) \approx\left[\left(2 X^{1 / 2} / f\right)-\left(1 / f^{2}\right)\right]+4\left[k r-(k a) f^{1 / 2} X^{1 / 4}\right]^{2} /(k a f)^{2}
$$

is locally parabolic. The first term in Eq. (30) is a constant baseline, and the second term is a harmonic oscillator potential centered on the position $r=a f^{1 / 2} X^{1 / 4}$. When the partial wave is at the high end of the edge region, $X$ is slightly less than $\left[\left(f^{2}+1\right) / 2 f\right]^{2}$ in Eq. (26), the well is very shallow, and its center is approximately halfway between the two classical turning points of Eq. (27). When the partial wave is at the low end of the edge region, $X$ is slightly greater than 1 in Eq. (26), and the well becomes substantially wider and somewhat deeper. If this interior well becomes deep enough, it can support one or more harmonic oscillator bound states that damp to zero as $r \rightarrow \infty$ if Eq. (30) were valid all the way out to infinity. Instead, these states slowly decay as they leak through the centrifugal barrier of Eq. (28) to the classically allowed region outside the lens. These metastable states are the MDRs. Substituting Eq. (30) into Eq. (20), the resulting differential equation is identical to a one-dimensional Schrodinger equation with a harmonic oscillator potential. If $X$ takes on the special values

$$
X^{1 / 2}=\left[\left(f^{2}+1\right) / 2 f\right]-[(2 S+1) / k a],
$$

where $S=0,1,2,3 \ldots$, analogous to the energy eigenvalues of the Schrodinger equation, the differential equation has a bound state solution. Using the approximation

$$
n(n+1) \approx(n+1 / 2)^{2}
$$

for large partial waves, Eq. (31) becomes

$$
n+1 / 2=\left[\left(f^{2}+1\right) k a / 2 f\right]-(2 S+1)
$$

or

$$
k a=(n+2 S+3 / 2)\left[2 f /\left(f^{2}+1\right)\right] .
$$

Interestingly, Eq. (34) is also the condition for which the exact confluent hypergeometric radial function [15] in Eq. (18) becomes a polynomial of degree $S$, again giving a bound state solution to Eq. (17) that damps to zero as $r$ $\rightarrow \infty$. Apparently, the two approximations of Eqs. (30) and (32) cancel each other. Considering only the condition for the existence of a bound state in the locally parabolic well and neglecting small corrections to $k a$ due to the need for matching boundary conditions at both ends of the centrifugal barrier, Eq. (34) is the approximate size parameter for the formation of a TE-polarized MDR in the partial wave $n$ of radial order $S$. In [7] this prediction is numerically tested, and the TM resonant size parameters are obtained.

By way of comparison, for scattering by a homogeneous sphere with $n$ slightly larger than $k a$, the radial effective potential consists of a centrifugal barrier outside the particle and an approximately linearly decreasing well, i.e., an Airy well, just inside the particle surface that also supports TE and TM resonances. The size parameter of the homogeneous sphere resonances is given to first order by [16-18]

$$
\begin{aligned}
k a \approx & (n+1 / 2) / N+(n+1 / 2)^{1 / 3} w_{S} /\left(2^{1 / 3} N\right)-P /\left(N^{2}-1\right)^{1 / 2} \\
& +\ldots,
\end{aligned}
$$

where

$$
\begin{aligned}
& P=1 \quad \text { for } \mathrm{TE}, \\
& =1 / N^{2} \quad \text { for } \mathrm{TM},
\end{aligned}
$$

where $A i\left(-w_{S}\right)=0$ and $S=1,2,3 \ldots$ The modified Luneburg lens MDRs are expected to have somewhat of a different behavior than the homogeneous sphere MDRs. The locally parabolic effective potential of Eqs. (21) and (30) is relatively wide and shallow and thus supports a bound state quite close to the bottom of the well, whereas the energy of the first bound state in an Airy well lies higher above the bottom of the well. As a result, the modified Luneburg lens MDRs for a given partial wavenumber should occur at a lower value of $X$ than do the MDRs of a homogeneous sphere. Also, since the deepest part of the well for a modified Luneburg lens lies further inside the sphere than does the deepest part of the Airy well, the Luneburg lens MDRs should also lie deeper inside the sphere, whereas the homogeneous sphere MDRs lie just beneath the sphere surface. These issues are treated more quantitatively and fully in [7]. 


\section{PHYSICAL OPTICS MODEL OF SCATTERING OF THE ORBITING RAY BY A LUNEBURG LENS WITH $\boldsymbol{f}=\mathbf{1}$}

In ray theory the amplitude for scattering by a Luneburg lens with $f=1$ is proportional to $(\cos \theta)^{1 / 2}$ for $0 \leqslant \theta \leqslant \pi / 2$ and vanishes for larger angles. The slope of this amplitude has an infinite discontinuity at $\theta=\pi / 2$ and is known as a weak caustic [19]. This divergence is softened in wave theory, as is shown here using the physical optics model. Consider a plane wave incident on a spherical scatterer. The spatial density of trays associated with the plane wave is $E_{0}{ }^{2}$, and the wavefront at the entrance plane tangent to the sphere surface is flat. For scattering at what is called a generic angle $\theta$, the scattered wavefront in the exit plane tangent to the sphere surface centered on the ray exiting in the $\theta$ direction is approximately parabolic in both the horizontal and vertical directions, having the radii of curvature $R_{h}$ and $R_{v}$, respectively. As one follows the incident flux tube through the sphere, the density of outgoing rays in the horizontal and vertical directions in the exit plane is taken to be $A_{h} E_{0}$ and $A_{v} E_{0}$, respectively. Let the exit plane's horizontal direction be $x^{\prime}$ and its vertical direction be $y^{\prime}$. Let $b_{\theta}$ be the impact parameter of the incident ray that is transmitted through the Luneburg lens and exits at the angle $\theta$. Then for $f=1$, rays with incident impact parameters $b$ $<b_{\theta}$ are scattered through smaller angles and cross the exit plane at $x^{\prime}>0$. Incident rays with impact parameters $b>b_{\theta}$ are scattered through larger angles and cross the exit plane at $x^{\prime}<0$.

In the physical optics model [20], the far-zone scattered electric field at the scattering angle $\theta$ is obtained by Fraunhofer diffracting the electric field in the scatterer's exit plane

$$
\begin{aligned}
E(\theta)= & {\left[-i k E_{0} \exp (i k r) / 2 \pi r\right] A_{h} A_{v} \exp [i \Phi(\theta)] } \\
& \times \int_{-\infty}^{\infty} \mathrm{d} x^{\prime} \exp \left(i k x^{\prime 2} / 2 R_{h}\right) \int_{-\infty}^{\infty} \mathrm{d} y^{\prime} \exp \left(i k y^{\prime 2} / 2 R_{v}\right) \\
= & {\left[E_{0} \exp (i k r) / r\right] A_{h} A_{v}\left(R_{h} R_{v}\right)^{1 / 2} \exp [i \Phi(\theta)], }
\end{aligned}
$$

where $\Phi(\theta)$ is the phase of the wavefront at the center of the exit plane. For ray transmission through a Luneburg lens with $f=1$ and assuming $100 \%$ transmission through the lens for both the TE and TM polarizations, a ray tracing analysis gives, after much algebra,

$$
\begin{aligned}
R_{v}=R_{h} & =a[1-\cos (\theta)], \\
A_{h} & =\{\cos \theta /[1-\cos (\theta)]\}^{1 / 2}, \\
A_{v} & =\{1 /[1-\cos (\theta)]\}^{1 / 2}, \\
\Phi(\theta) & =k a[(\pi / 2)-\cos (\theta)]-\pi .
\end{aligned}
$$

As a check of Eqs. (38a)-(38d), the radii of curvature of the phase fronts at the exit plane are equal to the distance along the ray exiting in the $\theta$ direction from the point focus on the back of the Luneburg lens, since the focal point is the source of the phase fronts. Further, when Eqs. (38a)-(38c) are substituted into Eq. (37), the result agrees with the ray theory scattered field of Eq. 18 of [1]. Finally, Eq. (38d) was derived in [1].

This analysis encounters difficulties at the nongeneric angle $\theta=\pi / 2$ for two reasons. First, the density of outgoing rays in the horizontal direction goes to zero there. Second, ray theory predicts that no rays are scattered for $\theta$ $>\pi / 2$, so there are no transmitted rays to integrate over for the $-\infty<x^{\prime}<0$ portion of the exit plane corresponding to

$$
\theta=(\pi / 2)+\Delta,
$$

with $\Delta>0$. Ray scattering occurs only for $\theta<\pi / 2$, i.e., for $\Delta<0$, and these transmitted rays cross the exit plane for $0<x^{\prime}<\infty$. Both difficulties are remedied by the same prescription. The ray density factor $A_{h}$ is slowly varying at angles far from $\pi / 2$ and rapidly decreases only as $\theta$ $\rightarrow \pi / 2$ due to its infinite slope at $\theta=\pi / 2$. As a result, one can approximate $A_{h}$ by some constant average value $A_{h}{ }^{\text {ave }}$ for $0 \leqslant \theta \leqslant \pi / 2$ corresponding to rays that cross the exit plane for $0 \leqslant x^{\prime}<\infty$, and by zero for $\pi / 2 \leqslant \theta \leqslant \pi$ corresponding to the absence of transmitted rays crossing the exit plane for $-\infty<x^{\prime}<0$. One then obtains

$$
\begin{aligned}
E[(\pi / 2)+\Delta]= & {\left[-i k E_{0} \exp (i k r) / 2 \pi r\right] A_{h}{ }^{\text {ave }} A_{v} \exp [i \Phi(\pi / 2)] } \\
& \times \int_{-\infty}^{\infty} \mathrm{d} x^{\prime} \exp \left(i k x^{\prime 2} / 2 R_{h}\right) \exp \left(i k x^{\prime} \Delta\right) \\
& \times \int_{-\infty}^{\infty} \mathrm{d} y^{\prime} \exp \left(i k y^{\prime 2} / 2 R_{v}\right) \\
= & \left(1 / 2^{1 / 2} r\right)\left[E_{0} a A_{h}{ }^{\text {ave }} \exp (i k r+i \Phi(\pi / 2)-i \pi / 4)\right] \\
& \times \exp \left(-i k a \Delta^{2} / 2\right)\left\{F(\infty)-F\left[\Delta(k a / \pi)^{1 / 2}\right]\right\},
\end{aligned}
$$

where $F(w)$ is the Fresnel integral

$$
F(w)=\int_{0}^{W} \mathrm{~d} v \exp \left(i \pi v^{2} / 2\right) .
$$

In the ray theory illuminated region, $\Delta$ is negative and the Fresnel straight-edge pattern $F(\infty)-F\left[\Delta(k a / \pi)^{1 / 2}\right]$ is oscillatory. In the ray theory shadowed region, $\Delta$ is positive and $F(\infty)-F\left[\Delta(k a / \pi)^{1 / 2}\right]$ monotonically decreases. This smoothing of the transition from the illuminated region to the shadowed region in the vicinity of $\theta=\pi / 2$ is shown in Fig. 5 of [11] for $k a=60.0$, and in Fig. 2 here for $k a=350.0$.

These results have a pleasing physical interpretation. In ray theory the orbiting ray travels in a circular arc on the surface of the lens and exits only at $\theta=\pi / 2$. In the physical optics model the orbiting ray travels along the surface of the lens forever, continually shedding radiation tangentially. The radiation it sheds for $\theta<\pi / 2$ interferes with the light transmitted through the sphere by incident rays with smaller impact parameters, producing an interference pattern. For $\theta>\pi / 2$, the shed radiation is the only contribution to the scattered light. As the amplitude of the orbiting ray decreases, the amount of radiation it sheds farther on in its trajectory decreases as well. This behavior is qualitatively similar to that of the radiation shed by electromagnetic surface waves into the shadowed 


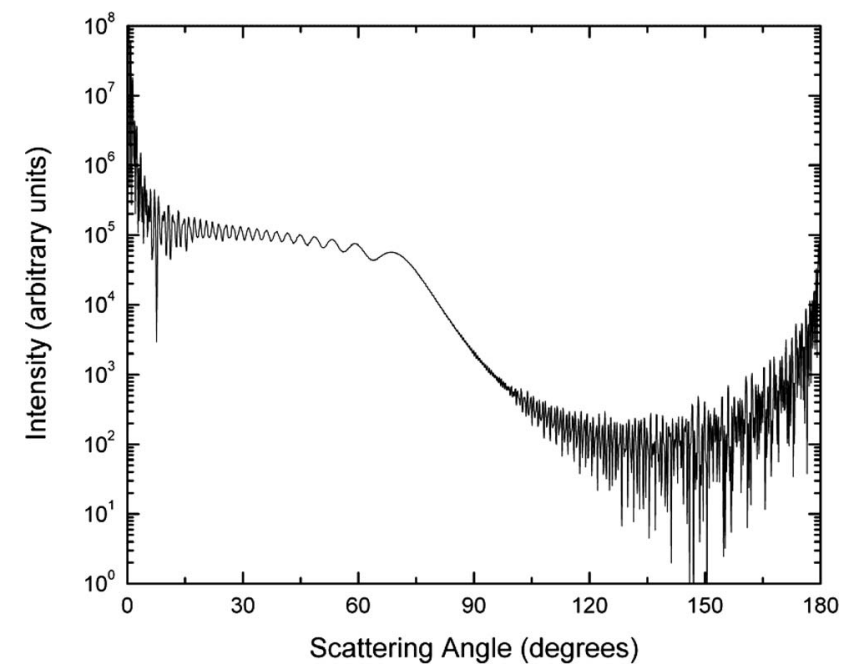

Fig. 2. TE scattered intensity as a function of the scattering angle $\theta$ for $f=1.0, a=28.40 \mu \mathrm{m}, \lambda=0.51 \mu \mathrm{m}$, and $k a=350.0 \mathrm{com}$ puted by the method described in [7]. For $30^{\circ}<\theta<100^{\circ}$, the intensity resembles that of a Fresnel straight-edge pattern corresponding to Eq. (40).

region at a Fock transition [21]. This analogy is only qualitative, however, since the angular dependence of the electromagnetic surface wave radiation damps exponentially in $\Delta$, whereas deep in the classically shadowed region here the falloff of Eq. (40) is proportional to $1 / \Delta$. In Fig. 2, the falloff of the intensity in the shadowed region was found to be better fit by a straight line using a log-log graph rather than a semilog graph, indicating a power law behavior. In addition, if one considers $f=1$ scattering as the limit of $f>1$ scattering, the $f>1$ rainbow angle approaches $\theta=\pi / 2$ and the rainbow electric field shifts from an Airy integral to the derivative of an Airy integral [1], thus suppressing the main rainbow peak. The supernumerary interference pattern of the $f>1$ rainbow, which occurs at smaller scattering angles than the main rainbow peak, evolves into the ripple pattern of the Fresnel straight-edge field of Eq. (40). The complex ray of the $f>1$ rainbow, which occurs at angles larger than the main rainbow peak, evolves into the smooth falloff of the radiation shed by the orbiting ray in the classically shadowed region. All these features are qualitatively evident in Fig. 2. The physical optics model of Eq. (40), however, provides only an approximation to the scattered field in the transition region. For $k a=350$, the main peak of the Fresnel straight-edge pattern should occur at $\theta=86.6^{\circ}$ according to Eq. (40). But in Fig. 2 it actually occurs at $\theta=68.8^{\circ}$. Similarly, the periodicity of the first number of oscillations observed in Fig. 2 for $\theta<68.8^{\circ}$ is a factor of 1.48 greater than the periodicity predicted from Eq. (40). As the sphere size parameter further increases toward the geometrical optics limit, these differences should slowly decrease.

Finally, in Fig. 2 the diffraction structure is evident for $\theta<10^{\circ}$, and the radiation shed by waves orbiting in all planes of incidence constructively interferes to form a broad glory enhancement in the transmitted intensity at $\theta \approx \pi$. The fine oscillatory structure for $\theta>100^{\circ}$ is due to interference of orbiting radiation shed by the ray with grazing incidence at the top of the sphere and the coun- terpropagating orbiting radiation shed by the ray with grazing incidence at the bottom of the sphere. All the effects qualitatively described here are treated quantitatively in [7] using a finely stratified multilayer sphere to model the modified Luneburg lens.

\section{APPENDIX A: ORBITING IN CLASSICAL SCATTERING}

In the context of classical mechanics, consider a spherically symmetric attractive potential $V(r)$ surrounding a force center located at the origin of coordinates. A particle of mass $m$ is incident on the potential from infinity. It has the impact parameter $b$ with respect to the force center, energy $E=\mathrm{mv}^{2}(\infty) / 2$, and angular momentum $L=\operatorname{mv}(\infty) b$, where $v(\infty)$ is the magnitude of the particle's velocity at infinity. The particle is deflected through an angle $\theta$ by the potential. The particle moves in the effective radial potential

$$
U_{\text {eff }}(r)=\left(L^{2} / 2 m r^{2}\right)+V(r)
$$

where the first term is the centrifugal potential and the second term is the attractive potential surrounding the force center. During the deflection of the particle, its distance of closest approach to the origin $r_{0}$ occurs when the radial kinetic energy vanishes and

$$
E=U_{\text {eff }}\left(r_{0}\right) \text {. }
$$

The deflection angle of the particle is [22]

$$
\theta=\pi-2 \int_{r_{0}}^{\infty}\left(L \mathrm{~d} r / r^{2}\right) /\left\{2 m\left[E-U_{\text {eff }}(r)\right]\right\}^{1 / 2} .
$$

For the generic situation where the particle arrives at $r_{0}$ and a positive radial force acts on it attempting to push it back out, one has

$$
\left.\left(\mathrm{d} U_{\text {eff }} / \mathrm{d} r\right)\right|_{r_{0}} \neq 0 .
$$

The integral in Eq. (A3) is convergent, the deflection angle is finite, and the particle spends a finite amount of time in the vicinity of the force center. For the nongeneric situation where the particle gets to $r_{0}$ and there is no radial force acting on it to push it back out, one has

$$
\left.\left(\mathrm{d} U_{\text {eff }} d \mathrm{~d} r\right)\right|_{r_{0}}=0 .
$$

The deflection angle of Eq. (A3) diverges logarithmically at the lower limit of integration [5,6]. The particle is captured by the force center in an orbit that slowly decays toward $r=r_{0}$, and it spends an infinite amount of time in the vicinity of the force center. Equation (A5) is the orbiting condition, and it corresponds to a particle whose energy is such that $U_{\text {eff }}$ is locally quadratic in the vicinity of $r_{0}$ and has a relative maximum there. Finally, if the potential is more slowly varying in the vicinity of $r_{0}$ so that

$$
\left.\left(\mathrm{d} U_{\text {eff }} / \mathrm{d} r\right)\right|_{r_{0}}=\left.\left(\mathrm{d}^{2} U_{\text {eff }} / \mathrm{d} r^{2}\right)\right|_{r_{0}}=0,
$$

the divergence of the deflection angle at the lower limit of integration in Eq. (A3) is stronger and the decay of the orbit toward $r=r_{0}$ is slower. Equation (A6) corresponds to a particle whose energy is such that the effective potential 
is locally cubic in the vicinity of $r_{0}$ and has its inflection point there.

\section{APPENDIX B. DEBYE SERIES FOR A SCATTERING BY A RADIALLY INHOMOGENEOUS SPHERE}

In this appendix the Debye series for scattering by a radially inhomogeneous sphere of radius $a$ with the arbitrary refractive index profile $N(r)$ in an external medium with refractive index $N=1$ is derived and compared with two different Debye series decompositions for a multilayer sphere approximating $N(r)$. The interaction of each incident partial wave with the radially inhomogeneous sphere is seen here to be decomposed into a sum of diffraction, external reflection from the sphere surface, and transmission through the sphere surface following $p$ -1 internal reflections from the surface with $p=1,2,3 \ldots$. This decomposition for a radially inhomogeneous sphere merits a number of comments. Whenever a wave is incident on an interface between two media with different refractive indices, part of the wave is transmitted through the interface and part is reflected by it. Thus as an incident wave penetrates into a radially inhomogeneous sphere, it continually sheds new reflected waves at every point along its trajectory. A portion of these reflected waves are in turn reflected over and over again as they encounter further refractive index changes while propagating through the sphere. The entire multiple-scattering infinite series of all the transmissions and reflections a partial wave can make before finally exiting the sphere results in the radial functions $F_{n}(k r)$ and $G_{n}(k r)$ of Eqs. (3) and (7) for the TE and TM polarizations. For scattering by a finely stratified multilayer sphere that approximates the refractive index profile $N(r)$, the individual terms of the multiple-scattering series described above were enumerated and studied in [23-25]. In the Debye series decomposition for an inhomogeneous sphere presented in this appendix, all the multiple-scattering interactions a partial wave makes within the sphere are summed implicitly, while only reflections and transmissions at the surface of the radially inhomogeneous sphere are counted explicitly. The derivation proceeds as follows.

Consider the partial wave radial functions for the TE polarization. Since Eq. (3) is a second-order differential equation, it has two linearly independent solutions. If $N(r)$ is finite both at the origin and at infinity, the wellbehaved solution at the origin is called $F_{n}(k r)$ and has the asymptotic behavior

$$
\begin{gathered}
\lim _{k r \rightarrow 0} F_{n}(k r) \sim(k r)^{n+1}, \\
\lim _{k r \rightarrow \infty} F_{n}(k r) \sim \cos \left[N(\infty) k r-\varphi_{n}\right],
\end{gathered}
$$

where $\varphi_{n}$ is a suitably chosen phase factor. The other linearly independent solution $U_{n}(k r)$ is singular at the origin and has the asymptotic behavior

$$
\lim _{k r \rightarrow 0} U_{n}(k r) \sim(k r)^{-n},
$$

$$
\lim _{k r \rightarrow \infty} U_{n}(k r) \sim \sin \left[N(\infty) k r-\varphi_{n}\right]
$$

These functions are radial standing waves. Let

$$
X_{n}^{(1 / 2)}(k r)=F_{n}(k r) \pm i U_{n}(k r)
$$

be the radially outgoing (1) and incoming (2) waves formed from these standing waves. Similarly, let the linearly independent solutions of the second-order differential equation of Eq. (7) for the TM polarization that are well behaved and singular at the origin be $G_{n}(k r)$ and $V_{n}(k r)$, where

$$
\begin{gathered}
\lim _{k r \rightarrow 0} G_{n}(k r) \sim(k r)^{n+1}, \\
\lim _{k r \rightarrow \infty} G_{n}(k r) \sim \cos \left[N(\infty) k r-\varphi_{n}\right], \\
\lim _{k r \rightarrow 0} V_{n}(k r) \sim(k r)^{-n}, \\
\lim _{k r \rightarrow \infty} V_{n}(k r) \sim \sin \left[N(\infty) k r-\varphi_{n}\right],
\end{gathered}
$$

and where we have additionally assumed that $(d N / d r)_{0}$ $=0$. Again let

$$
Z_{n}^{(1,2)}(k r)=G_{n}(k r) \pm i V_{n}(k r)
$$

be the radially outgoing (1) and incoming (2) traveling waves formed from these standing waves. For scattering by a homogeneous sphere of refractive index $N$, the functions $F_{n}$ and $G_{n}, U_{n}$ and $V_{n}$, and $X_{n}{ }^{(1,2)}$ and $Z_{n}^{(1,2)}$ become Riccati-Bessel, Riccati-Neumann, and Riccati-Hankel functions of $N k r$, respectively.

In analogy to the treatment of [23], let the amplitudes $N_{n}, D_{n}, P_{n}$, and $Q_{n}$ for the TE polarization be defined as

$$
\begin{aligned}
& N_{n}=F_{n}{ }^{\prime}(k a) \psi_{n}(k a)-F_{n}(k a) \psi_{n}{ }^{\prime}(k a), \\
& D_{n}=F_{n}{ }^{\prime}(k a) \chi_{n}(k a)-F_{n}(k a) \chi_{n}{ }^{\prime}(k a), \\
& P_{n}=U_{n}^{\prime}(k a) \psi_{n}(k a)-U_{n}(k a) \psi_{n}^{\prime}(k a), \\
& Q_{n}=U_{n}{ }^{\prime}(k a) \chi_{n}(k a)-U_{n}(k a) \chi_{n}{ }^{\prime}(k a) .
\end{aligned}
$$

The corresponding amplitudes for the TM polarization are obtained by replacing $F_{n}(k a)$ and $U_{n}(k a)$ with $N^{2}(a) G_{n}(k a)$ and $N^{2}(a) V_{n}(k a)$, and by replacing $F_{n}{ }^{\prime}(k a)$ and $U_{n}{ }^{\prime}(k a)$ with $G_{n}{ }^{\prime}(k a)$ and $V_{n}{ }^{\prime}(k a)$, where $N^{2}(a)$ is evaluated inside the sphere as $r \rightarrow a$. The Wronskian of $X_{n}{ }^{(1)}$ and $X_{n}{ }^{(2)}$ and of $Z_{n}{ }^{(1)}$ and $Z_{n}{ }^{(2)}$ is defined as

$$
W_{n}{ }^{\mathrm{TE}}(k a)=i\left[X_{n}^{(1)}(k a) X_{n}^{(2) \prime}(k a)-X_{n}^{(1) \prime}(k a) X_{n}^{(2)}(k a)\right] / 2,
$$

$$
W_{n}{ }^{\mathrm{TM}}(k a)=i\left[Z_{n}{ }^{(1)}(k a) Z_{n}{ }^{(2) \prime}(k a)-Z_{n}{ }^{(1) \prime}(k a) Z_{n}^{(2)}(k a)\right] / 2 .
$$

These Wronskians are found to satisfy

$$
W_{n}^{\mathrm{TE}}=N_{n} Q_{n}-D_{n} P_{n},
$$




$$
N^{2}(a) W_{n}^{\mathrm{TM}}=N_{n} Q_{n}-D_{n} P_{n} .
$$

Consider the interior of the radially inhomogeneous sphere to be medium 1 and the exterior to be medium 2 . The partial wave transmission and reflection coefficients at the surface of the radially inhomogeneous sphere for both polarizations are derived as follows. When a radially incoming partial wave in medium 2 with the radial function $\zeta_{n}{ }^{(2)}(k r)$ is incident on the surface of the radially inhomogeneous sphere, a portion $T_{n}{ }^{21}$ is transmitted at the surface of the sphere with the radial function $X_{n}^{(2)}(k r)$ or $Z_{n}{ }^{(2)}(k r)$, and a portion $R_{n}{ }^{22}$ is reflected from the surface back into medium 2 with the radial function $\zeta_{n}{ }^{(1)}(k r)$. Matching the boundary conditions of the various components of $\mathbf{E}$ and $\mathbf{B}$ at the surface of the sphere gives

$$
\begin{aligned}
T_{n}{ }^{21}= & -2 i /\left[X_{n}{ }^{(2) \prime}(k a) \zeta_{n}{ }^{(1)}(k a)-X_{n}{ }^{(2)}(k a) \zeta_{n}{ }^{(1) \prime}(k a)\right], \\
R_{n}{ }^{22}= & -\left[X_{n}{ }^{(2) \prime}(k a) \zeta_{n}{ }^{(2)}(k a)-X_{n}{ }^{(2)}(k a) \zeta_{n}{ }^{(2) \prime}(k a)\right] / \\
& {\left[X_{n}{ }^{(2) \prime}(k a) \zeta_{n}{ }^{(1)}(k a)-X_{n}{ }^{(2)}(k a) \zeta_{n}{ }^{(1) \prime}(k a)\right] }
\end{aligned}
$$

for the TE polarization and

$$
\begin{aligned}
T_{n}{ }^{21}= & -2 i N^{2}(a) /\left[Z_{n}{ }^{(2) \prime}(k a) \zeta_{n}{ }^{(1)}(k a)-N^{2}(a)\right. \\
& \left.\times Z_{n}{ }^{(2)}(k a) \zeta_{n}{ }^{(1) \prime}(k a)\right] \\
R_{n}{ }^{22}= & -\left[Z_{n}{ }^{(2) \prime}(k a) \zeta_{n}{ }^{(2)}(k a)-N^{2}(a) Z_{n}{ }^{(2)}(k a) \zeta_{n}{ }^{(2) \prime}(k a)\right] / \\
& {\left[Z_{n}{ }^{(2) \prime}(k a) \zeta_{n}{ }^{(1)}(k a)-N^{2}(a) Z_{n}{ }^{(2)}(k a) \zeta_{n}{ }^{(1) \prime}(k a)\right] }
\end{aligned}
$$

for the TM polarization. For both polarizations, the transmission and reflection coefficients are of the form

$$
\begin{aligned}
T_{n}^{21}= & -2 i \sigma /\left[\left(N_{n}+Q_{n}\right)+i\left(D_{n}-P_{n}\right)\right], \\
R_{n}{ }^{22}= & {\left[\left(-N_{n}+Q_{n}\right)+i\left(D_{n}+P_{n}\right)\right] /\left[\left(N_{n}+Q_{n}\right)\right.} \\
& \left.+i\left(D_{n}-P_{n}\right)\right], \\
\sigma= & 1 \quad \text { for } \mathrm{TE}, \\
= & N^{2}(a) \quad \text { for TM. }
\end{aligned}
$$

Similarly, when a radially outgoing partial wave in medium 1 with the radial function $X_{n}^{(1)}(k r)$ or $Z_{n}^{(1)}(k r)$ is incident on the surface of the radially inhomogeneous sphere, a portion $T_{n}{ }^{12}$ is transmitted at the surface to the outside with the radial function $\zeta_{n}{ }^{(1)}(k r)$, and a portion $R_{n}{ }^{11}$ is reflected from the surface back inside with the radial function $X_{n}{ }^{(2)}(k r)$ or $Z_{n}{ }^{(2)}(k r)$. Boundary condition matching at the surface of the sphere gives

$$
T_{n}{ }^{12}=-2 i W_{n}{ }^{\mathrm{TE}} /\left[X_{n}{ }^{(2) \prime}(k a) \zeta_{n}{ }^{(1)}(k a)-X_{n}{ }^{(2)}(k a) \zeta_{n}{ }^{(1) \prime}(k a)\right],
$$

$$
\begin{aligned}
& R_{n}{ }^{11}=-\left[X_{n}{ }^{(1) \prime}(k a) \zeta_{n}{ }^{(1)}(k a)-X_{n}{ }^{(1)}(k a) \zeta_{n}{ }^{(1) \prime}(k a)\right] / \\
& {\left[X_{n}^{(2) \prime}(k a) \zeta_{n}{ }^{(1)}(k a)-X_{n}{ }^{(2)}(k a) \zeta_{n}{ }^{(1) \prime}(k a)\right]}
\end{aligned}
$$

for the TE polarization and

$$
\begin{aligned}
T_{n}{ }^{12}= & -2 i W_{n}{ }^{\mathrm{TM}} /\left[Z_{n}{ }^{(2) \prime}(k a) \zeta_{n}{ }^{(1)}(k a)-N^{2}(a)\right. \\
& \left.\times Z_{n}{ }^{(2)}(k a) \zeta_{n}{ }^{(1) \prime}(k a)\right], \\
R_{n}{ }^{11}= & -\left[Z_{n}{ }^{(1) \prime}(k a) \zeta_{n}{ }^{(1)}(k a)-N^{2}(a) Z_{n}{ }^{(1)}(k a) \zeta_{n}{ }^{(1) \prime}(k a)\right] / \\
& {\left[Z_{n}{ }^{(2) \prime}(k a) \zeta_{n}{ }^{(1)}(k a)-N^{2}(a) Z_{n}{ }^{(2)}(k a) \zeta_{n}{ }^{(1) \prime}(k a)\right] }
\end{aligned}
$$

for the TM polarization. For both polarizations, the transmission and reflection coefficients are of the form

$$
\begin{aligned}
T_{n}{ }^{12}= & -2 i W_{n} /\left[\left(N_{n}+Q_{n}\right)+i\left(D_{n}-P_{n}\right)\right], \\
R_{n}{ }^{11}= & {\left[\left(-N_{n}+Q_{n}\right)-i\left(D_{n}+P_{n}\right)\right] /\left[\left(N_{n}+Q_{n}\right)\right.} \\
& \left.+i\left(D_{n}-P_{n}\right)\right] .
\end{aligned}
$$

The partial wave scattering amplitudes can then be shown, after a reasonable amount of algebra, to be of the form

$$
\begin{aligned}
& \left.\begin{array}{l}
a_{n} \\
b_{n}
\end{array}\right\}=N_{n} /\left(N_{n}+i D_{n}\right) \\
& =\left(\frac{1}{2}\right)\left[1-R_{n}{ }^{22}-T_{n}{ }^{21} T_{n}{ }^{12} /\left(1-R_{n}{ }^{11}\right)\right] \\
& =\left(\frac{1}{2}\right)\left[1-R_{n}{ }^{22}-\sum_{p=1}^{\infty} T_{n}{ }^{21}\left(R_{n}{ }^{11}\right)^{p-1} T_{n}{ }^{12}\right] .
\end{aligned}
$$

This is the Debye series expansion for a radially inhomogeneous sphere. The first term on the right-hand side of Eq. (B16) represents diffraction of the plane wave and is independent of the composition of the sphere. Thus, this term is identical for both a homogeneous sphere and a modified Luneburg lens. The second term is external reflection from the surface of the radially inhomogeneous sphere. Whenever a wave reflects from a surface, the reflection amplitude depends on the details of the material the wave is reflecting from, even though the reflected wave never enters the material. This dependence is described by the interior functions $X_{n}{ }^{(2)}$ and $Z_{n}{ }^{(2)}$ evaluated at the surface of the sphere in Eqs. (B10b) and (B11b). The last term in Eq. (B16) represents transmission through the sphere following $p-1$ internal reflections at the surface. For example, the term $T_{n}{ }^{21} T_{n}{ }^{12}$ corresponds to a partial wave crossing the sphere surface, making an infinite series of transmissions and reflections inside, and when encountering the sphere surface for the second time, is transmitted out. Similarly, the term $T_{n}{ }^{21} R_{n}{ }^{11} T_{n}{ }^{12}$ in Eq. (B16) corresponds to a partial wave crossing the sphere surface, making an infinite series of reflections and transmissions inside the sphere, eventually returning to the surface where it is reflected back inside, making another infinite series of transmissions and reflec- 
tions, arriving at the surface again and being transmitted out. A similar calculation of the partial wave interior amplitudes gives the Debye series decomposition

$$
\begin{aligned}
& \left.\begin{array}{l}
c_{n} \\
d_{n}
\end{array}\right\}=-i \sigma /\left(N_{n}+i D_{n}\right)=T_{n}{ }^{21} /\left(1-R_{n}{ }^{11}\right) \\
& =\sum_{p=1}^{\infty} T_{n}^{21}\left(R_{n}^{11}\right)^{p-1} .
\end{aligned}
$$

The meaning of the terms in Eq. (B16) is clarified by considering the situation of a multilayer sphere, as in [23-25], which approximates a radially inhomogeneous sphere. The infinite series of transmissions and reflections inside the multilayer sphere was decomposed in these references into a sequence of single-scattering transmission and reflection amplitudes evaluated at each interface. Each of these were expressed in terms of the Riccati-Hankel functions $\zeta_{n}\left(N_{j} k a_{j, j+1}\right)$ and $\zeta_{n}\left(N_{j+1} k a_{j, j+1}\right)$ for the constant refractive indices $N_{j}$ and $N_{j+1}$ to either side of the $j, j+1$ interface at the radius $a_{j, j+1}$. In Eq. (B16) the summation of this infinite series is implicit in the approximation of the smoothly joined Riccati-Hankel functions inside each layer of the multilayer sphere to the radially inhomogeneous sphere functions $X_{n}$ and $Z_{n}$.

In general, one may ask what it is that makes the Debye series decomposition of the partial wave scattering and interior amplitudes special, since there are an infinite number of ways to decompose $a_{n}$ and $b_{n}$ for a homogeneous sphere into the sum of either a finite or an infinite number of parts. Whether a decomposition is useful or not is determined by whether the individual parts either have an intuitive physical interpretation or simplify the numerical computation of the full amplitudes. The Debye series is meaningful because of the straightforward physical interpretibility of each of the individual terms. The situation is more complicated for the partial wave scattering amplitudes of a multilayer sphere, since many different Debye series decompositions are possible. The Debye series of [23] is not especially closely connected with the Debye series of Eqs. (B10)-(B16) for a radially inhomogeneous sphere. But an alternate Debye series for a multilayer sphere can be constructed that is closer in spirit to Eq. (B16).

Before this alternate Debye series is derived in this appendix, part of the notation and results of [23] must be briefly recounted as follows. The partial wave numbers and polarization states are suppressed, and one assumes a sphere is composed of $M$ concentric layers with a constant refractive index in each layer. The innermost layer is region 1 , the outermost layer is region $M$, and the external medium is region $M+1$. In [23] multiple-scattering amplitudes $T_{M+1,1} T_{1, M+1}, R_{M+1, \Gamma, M+1}$ and $R_{1, \Gamma, 1}$ were defined. For example $R_{M+1, \Gamma, M+1}$ corresponds to a partial wave crossing the $M, M+1$ interface (i.e., the sphere surface), making an infinite series of transmissions and reflections inside the sphere (denoted by $\Gamma$ ) without ever being transmitted into region 1 , crossing back out through the $M, M+1$ interface, and propagating out to infinity. This can be taken to be the multiple-scattering generalization of external reflection where a partial wave penetrates a certain depth into the sphere before turning around and being reflected out. While inside the multilayer sphere, the partial wave can internally reflect from either the sphere surface or the 1,2 interface any number of times before finally being transmitted out through the sphere surface. In [23], the partial wave scattering amplitudes of the $M$ layer sphere were written in terms of these multiple-scattering transmission and reflection amplitudes as the Debye series

$$
\left.\begin{array}{l}
a_{12 \ldots M+1} \\
b_{12 \ldots M+1}
\end{array}\right\}=\frac{1}{2}\left[1-R_{M+1, \Gamma, M+1}-T_{M+1,1} T_{1, M+1} /\left(1-R_{1, \Gamma, 1}\right)\right] .
$$

A prescription was also sketched there and is described in more detail in the next paragraph for relating the multiple-scattering transmission and reflection amplitudes to a series of single-scattering transmission and reflection amplitudes at the $j, j+1$ interface, which we denote here by $t_{j+1, j}, t_{j, j+1}, r_{j+1, j, j+1}$, and $r_{j, j+1, j}$.

The alternate Debye series decomposition of the scattering amplitudes for a multilayer sphere derived in this appendix considers the $M, M+1$ interface as special and explicitly describes every interaction of a partial wave with the sphere surface. As a first step in this alternate decomposition, the prescription for relating multiplescattering transmission and reflection amplitudes to individual-interface transmission and reflection amplitudes sketched in [23] is more explicitly given as follows. In the spirit of the progressive iteration scheme [26], the $M$ layer sphere multiple-scattering transmission and reflection amplitudes can be written in terms of the $M-1$ layer sphere multiple-scattering transmission and reflection amplitudes and the $M, M+1$ interface singlescattering amplitudes. After a reasonable amount of algebra, the result is

$$
\begin{aligned}
T_{M+1,1}= & t_{M+1, M} T_{M, 1} /\left(1-R_{M, \gamma, M} r_{M, M+1, M}\right), \\
T_{1, M+1}= & T_{1, M} t_{M, M+1} /\left(1-R_{M, \gamma, M} r_{M, M+1, M}\right), \\
R_{M+1, \Gamma, M+1}= & r_{M+1, M, M+1}+t_{M+1, M} R_{M, \gamma, M} t_{M, M+1} / \\
& \left(1-R_{M, \gamma, M} r_{M, M+1, M}\right), \\
R_{1, \Gamma, 1}= & R_{1, \gamma, 1}+T_{1, M} r_{M, M+1, M} T_{M, 1} / \\
& \left(1-R_{M, \gamma, M} r_{M, M+1, M}\right),
\end{aligned}
$$

where $\Gamma$ indicates multiple scattering within the entire $M$ layer sphere and $\gamma$ indicates multiple scattering within the first $M-1$ layers of the sphere. In this way individual layers of the multilayer sphere can be peeled away one by one, allowing the multiple-scattering amplitudes to be written as a sequence of individual interactions at all the interfaces.

The alternate Debye series for the multilayer sphere can now be obtained as follows. Let

$$
I_{M}=R_{M, \gamma, M}+T_{M, 1} T_{1, M} /\left(1-R_{1, \gamma, 1}\right)
$$

be the interaction of the partial wave with the first $M-1$ layers of the sphere so that the Debye series for the $M$ -1 layer sphere is 


$$
\left.\begin{array}{l}
a_{12 \ldots M} \\
b_{12 \ldots M}
\end{array}\right\}=\left(\begin{array}{l}
\frac{1}{2}
\end{array}\right)\left(1-I_{M}\right)
$$

in analogy with Eq. (B18) for the $M$ layer sphere. Then substituting Eqs. (B19) and (B20) into Eq. (B18) gives, after much algebra,

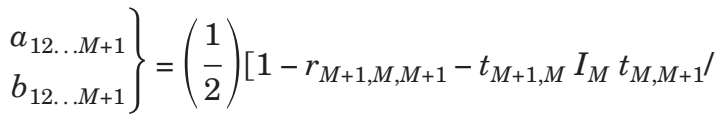

$$
\begin{aligned}
& \left.\left(1-I_{M} r_{M, M+1, M}\right)\right] \text {. }
\end{aligned}
$$

In Eq. (B22) each interaction of the partial wave with the $M, M+1$ interface (i.e., the sphere surface) is counted individually, and the interactions with all the interior interfaces are lumped together into the multiple-scattering amplitude $I_{M}$. This is the generalization of Eqs. (37) and (38) of [27] for the Debye series decomposition for a coated sphere. It is also closer in spirit to the radially inhomogeneous sphere Debye series of Eq. (B16) than is the original multilayer sphere Debye series of Eq. (B18), since the functions appearing in the reflection and transmission amplitudes of Eqs. (B10), (B11), (B13), and (B14) are evaluated only at the sphere surface.

For a modified Luneburg lens, where $N(a)=1$ inside the sphere as $r \rightarrow a$, one can expect that $r_{M+1, M, M+1}=r_{M, M+1, M}$ $=0$, since there is no refractive index step at the sphere surface. Thus

$$
\left.\begin{array}{l}
a_{12 \ldots M+1} \\
b_{12 \ldots M+1}
\end{array}\right\}=\left(\begin{array}{l}
\frac{1}{2} \\
2
\end{array}\right)\left(1-t_{M+1, M} I_{M} t_{M, M+1}\right),
$$

and the only contribution to nondiffractive scattering for the Luneburg lens is transmission in and out through the surface, accompanied by multiple scattering at all points inside.

\section{REFERENCES}

1. J. A. Lock, "Scattering of an electromagnetic plane wave by a Luneburg lens. I. Ray theory," J. Opt. Soc. Am. A 25, 2971-2979 (2008).

2. G. Arfken, Mathematical Methods for Physicists, 3rd ed. (Academic, 1985), p. 756, Eqs. (13.149) and (13.150).

3. M. Abramowitz and I. A. Stegun, Handbook of Mathematical Functions (National Bureau of Standards, 1964), p. 505, Eqs. (13.1.31) and (13.1.32).

4. D. J. Griffiths, Introduction to Quantum Mechanics, 2nd ed. (Pearson, 2005), pp. 68, 69, and 316.

5. K. W. Ford and J. A. Wheeler, "Semiclassical description of scattering," Ann. Phys. (N.Y.) 7, 259-286 (1959).

6. M. V. Berry and K. E. Mount, "Semiclassical approximations in wave mechanics," Rep. Prog. Phys. 35, 315-397 (1972), Sec. 6.3.

7. J. A. Lock, "Scattering of an electromagnetic plane wave by a Luneburg lens. III. Finely stratified sphere model," J. Opt. Soc. Am. A 25, 2991-3000 (2008).

8. P. J. Wyatt, "Scattering of electromagnetic plane waves from inhomogeneous spherically symmetric objects," Phys. Rev. 127, 1837-1843 (1962).

9. P. J. Wyatt, "Scattering of electromagnetic plane waves from inhomogeneous spherically symmetric objects (errata)," Phys. Rev. 134, AB1 (1964).

10. M. Kerker, The Scattering of Light and Other Electromagnetic Radiation (Academic, 1969), pp. 233-235.

11. B. R. Johnson, "Light scattering by a multilayer sphere," Appl. Opt. 35, 3286-3296 (1996).

12. C.-T. Tai, Dyadic Green's Functions in Electromagnetic Theory (Intext, 1971), pp. 201-204.

13. H. M. Nussenzveig, Diffraction Effects in Semiclassical Scattering (Cambridge U. Press, 1992), Chap. 14.

14. F. Michel, G. Reidenmeister, and S. Ohkubo, "Luneburg lens approach to nuclear rainbow scattering," Phys. Rev. Lett. 89, 152701-1-152704-4 (2002).

15. M. Abramowitz and I. A. Stegun, Handbook of Mathematical Functions (National Bureau of Standards, 1964), p. 504, Eqs. (13.1.2) and (13.1.3).

16. C. C. Lam, P. T. Leung, and K. Young, "Explicit asymptotic formulas for the positions, widths, and strengths of resonances in Mie scattering," J. Opt. Soc. Am. B 9, 1585-1592 (1992).

17. S. Schiller, "Asymptotic expansion of morphological resonance frequencies in Mie scattering," Appl. Opt. 32, 2181-2185 (1993).

18. B. R. Johnson, "Theory of morphology-dependent resonances: shape resonances and width formulas," J. Opt. Soc. Am. A 10, 343-352 (1993).

19. N. Fiedler-Ferrari, H. M. Nussenzveig, and W. J. Wiscombe, "Theory of near-critical-angle scattering from a curved interface," Phys. Rev. A 43, 1005-1038 (1991).

20. P. L. Marston, "Geometrical and catastrophe optics methods in scattering," Phys. Acoust. 21, 1-234 (1992), Sec. 2.6.

21. H. M. Nussenzveig, Diffraction Effects in Semiclassical Scattering (Cambridge U. Press, 1992), p. 59.

22. J. B. Marion and S. T. Thornton, Classical Dynamics of Particles and Systems, 3rd ed. (Harcourt Brace Jovanovich, 1988), p. 256, Eq. (7.38).

23. J. A. Lock, "Debye series analysis of scattering of a plane wave by a spherical Bragg grating," Appl. Opt. 44, 5594-5603 (2005).

24. R. Li, X. Han, H. Jiang, and K. F. Ren, "Debye series for light scattering by a multilayered sphere," Appl. Opt. 45, 1260-1270 (2006).

25. R. Li, X. Han, H. Jiang, and K. F. Ren, "Debye series of normally incident plane-wave scattering by an infinite multilayered cylinder," Appl. Opt. 45, 6255-6262 (2006).

26. W. C. Chew, Waves and Fields in Inhomogeneous Media (Van Nostrand, 1990), pp. 192-193.

27. J. A. Lock, J. M. Jamison, and C.-Y. Lin, "Rainbow scattering by a coated sphere," Appl. Opt. 33, 4677-4690, (1994). 